CALT-68-2148

hep-th/9712013

December 1997

\title{
Short Distance Contributions to Graviton-Graviton Scattering: Matrix Theory versus Supergravity
}

\author{
Esko Keski-Vakkuri ${ }^{1}$ and Per Kraus ${ }^{2}$ \\ California Institute of Technology \\ Pasadena CA 91125, USA \\ email: esko or perkraus@theory.caltech.edu
}

\begin{abstract}
:
We study graviton scattering in the presence of higher dimensional operators - particularly, $\mathcal{R}^{4}$ - arising from loop effects. We find that the results do not correspond to any known terms in the effective action of Matrix Theory, thus lending support to the idea that the finite N Matrix Theory has no simple relation to supergravity with large compactification radii.
\end{abstract}

${ }^{1}$ Work supported in part by a DOE grant DE-FG03-92-ER40701.

${ }^{2}$ Work supported in part by a DOE grant DE-FG03-92-ER40701 and by the DuBridge Foundation. 


\section{Introduction}

Matrix Theory [1] has been successful in reproducing certain features of classical, long distance supergravity in eleven dimensions [2]. Graviton scattering has been checked at the level of one and two graviton exchange [3, 1, 田, 5], including the leading order spin dependence [6, 7, 8]. Scattering processes involving extended branes have also been considered, and such processes have found a unified description in terms of the Matrix gauge theory [9, [10].

What has not been probed, however, is the short distance structure of supergravity. Viewing supergravity as a low energy effective field theory, the short distance structure is encoded by the presence of higher dimensional operators whose coefficients are suppressed by inverse powers of the Planck mass. The precise values of the coefficients are not calculable within the framework of the low energy theory; such a determination requires a fundamental description which is valid at short distances. Matrix Theory purports to be such a description, and so in principle allows one to compute the precise form of the low energy effective action.

Independent of Matrix Theory, there are certain higher dimensional operators in supergravity whose coefficients are known. One loop results in string theory [11], along with duality symmetries [12, 13, 14], strongly support the appearance of the term [12]

$$
\mathcal{L}_{\mathcal{R}^{4}}=\frac{\pi^{2}}{9 \cdot 2^{7} \cdot \kappa^{2 / 3}} \int d^{11} x \sqrt{g} t_{8} t_{8} \mathcal{R}^{4}
$$

where the operator $t_{8} t_{8} \mathcal{R}^{4}$ is defined in (24) as a particular contraction of four Riemann tensors. From the eleven dimensional point of view, the operator arises from a one loop diagram with short distance cutoff of order the eleven dimensional Planck length.

The term above introduces new vertices which contribute to graviton-graviton scattering. For instance, there is a new four point vertex which can contribute through the diagram in Fig. 1.

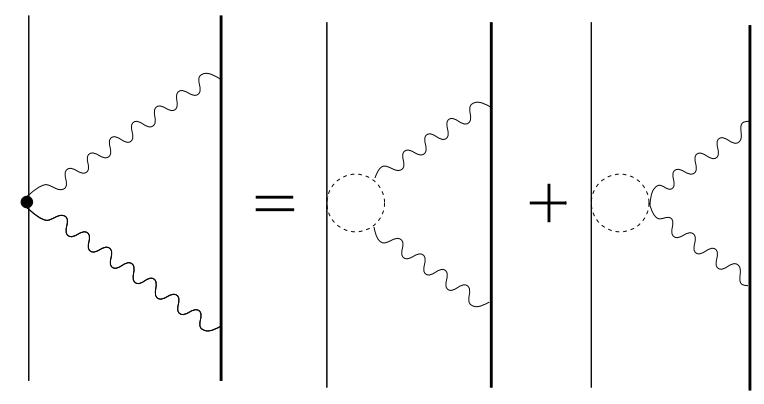

Figure 1: Contribution to graviton-graviton scattering. The thick and thin lines represent the source and probe gravitons respectively (see text), and the black dot in the leftmost diagram represents the $t_{8} t_{8} \mathcal{R}^{4}$ operator. The two diagrams on the right hand side illustrate the origin of $t_{8} t_{8} \mathcal{R}^{4}$ as arising from the divergent part of one loop diagrams. The dotted lines represent gravitons, anti-symmetric tensors, and gravitinos circulating in the loop. 
In sect. 3 we analyze this process by performing a generalized source-probe calculation. The result is expressed in terms of the effective action of the probe, and takes the form

$$
L \sim \frac{N_{s}^{2} N_{p}^{3} v^{8}}{M^{24} R^{7} r^{18}},
$$

where $M$ is the eleven dimensional Planck mass, $N_{p}, N_{s}$ are the longitudinal momenta of the gravitons according to $p_{-}=N / R$, and $v$ and $r$ are the relative transverse velocity and separation.

On the Matrix Theory side, the effective action for the probe has the structure

$$
L_{l}=R M^{6-3 l} r^{4-3 l} f_{l}\left(\frac{v}{R M^{3} r^{2}}\right)
$$

where $L_{l}$ denotes the contribution from $l$ loop diagrams. A particular two loop contribution is

$$
L_{2} \sim \frac{v^{8}}{M^{24} R^{7} r^{18}}
$$

which appears to match onto the term in (2). However, an $l$ loop diagram in Matrix Theory can give at most $l+1$ powers of $N$. Thus the supergravity contribution (2), which has five powers of $N$, cannot be reproduced from a two loop diagram in Matrix Theory. In fact, we see that there is no term in the expansion (3) which can reproduce the supergravity result.

The appearance of the troublesome $N_{p}$ dependence in (2) is easy to understand by considering the structure of the $\mathcal{R}^{4}$ operator. $\mathcal{R}^{4}$ has eight derivatives, and these act on the source and probe variables. The fact that (2) goes as $r^{-18}$ means that four of the derivatives are acting on the source, since two powers of the graviton propagator go as $r^{-14}$ if no derivatives act on them. The remaining four derivatives act on the probe; each derivative yields a factor of $N_{p}$, giving altogether $N_{p}^{4}$. Finally, one has to divide by $p_{-} \sim N_{p}$ to obtain the proper normalization of states in the light cone frame (or as is seen from the analysis in sect. 3). This results in the $N_{p}^{3}$ dependence of (2). What is more difficult to see is the numerical coefficient which multiplies the term in (2), and if it should happen to vanish then agreement with Matrix Theory would be reached. Therefore we are motivated to carefully compute this coefficient in sect. 3. We find that it is nonzero, indicating disagreement.

Recently, there have appeared arguments [15] which offer a derivation of the equivalence between Matrix Theory and supergravity with lightlike compactification. However, as discussed in [16], these arguments do not automatically apply to the regime of supergravity which is being considered in this paper, namely large compactification radii. Thus discrepancies between finite $N$ Matrix Theory [17] and the usual notion of supergravity cannot be ruled out, and indeed such discrepancies have already appeared [18].

The remainder of this paper is organized as follows. In sect. 2 we review the sourceprobe calculation of [5], point out its limits of validity, and show how it can be generalized. We derive the effect of the $t_{8} t_{8} \mathcal{R}^{4}$ operator in sect. 3 and discuss the sorts of terms in the probe action which can be generated. The calculation is somewhat involved due to 
the fact that the effect we are looking at is polarization dependent, and so the spin of the gravitons cannot be neglected even at leading order. In sect. 4 we discuss the accuracy of our approach and elaborate on the roles of the anti-symmetric tensor and gravitino, which have not appeared explicitly in our calculations. Finally, we comment on possible resolutions of the discrepancies.

\section{Graviton scattering in supergravity}

We begin by reviewing some results from previous work on graviton-graviton scattering. Ref. [5] studied the scattering of two gravitons in eleven dimensional supergravity with lightlike compactification. The null coordinate $x^{-}=x^{11}-t$ is taken to have periodicity $2 \pi R$. The lightlike momentum $p_{-}$is quantized in integer units of $1 / R$, so $p_{-}=N_{s} / R, N_{p} / R$ for the two gravitons. It is further assumed that $N_{s}$ is large enough that the first graviton can be considered to be a classical source, with the second graviton probing its gravitational field. That is, the scattering has to be soft enough so that momentum transfer is negligible and there are no recoil effects. The gravitational field of the source is given by the metric

$$
d s_{\mathrm{bg}}^{2}=-2 d \tau d x^{-}-h_{--}\left(d x^{-}\right)^{2}-d x_{\perp}^{2},
$$

where $\tau=x^{+} / 2=\left(x^{11}+t\right) / 2$ and $x_{\perp}^{i}$ are the 9 transverse coordinates. The graviton is located at $x_{\perp}=0$. A graviton with fixed $x^{-}$would produce the Aichelburg-Sexl metric [19]; however, here the graviton has a fixed lightlike momentum so the metric must be averaged over the lightlike circle. Upon averaging, the harmonic function $h_{--}$takes the form

$$
h_{--}=\frac{15 N_{s}}{2 R^{2} M^{9} r^{7}},
$$

where $r^{2} \equiv x_{\perp}^{2}, M$ is the Planck mass in eleven dimensions. From now on we will use the notation $h$ for the harmonic function $h_{--}$.

The authors of [5] find a point particle action for the probe graviton using the following strategy. They begin by writing the Lagrangian for a massive scalar particle moving in the background (5). Then they point out that the appropriate variational principle is one in which $p_{-}$is held fixed. Thus instead of using the Lagrangian one should use the Routhian, which is obtained by performing a Legendre transformation to eliminate $\dot{x}^{-}$in favor of $p_{-}$. Unlike the Lagrangian, the Routhian has a non-trivial limit as $m \rightarrow 0$. The (negative of the) Routhian is

$$
L^{\prime}=L-p_{-} \dot{x}^{-}=\left[-m \sqrt{g_{\mu \nu} \dot{x}^{\mu} \dot{x}^{\nu}}-p_{-} \dot{x}^{-}\right]_{m \rightarrow 0}=-p_{-} \dot{x}^{-}
$$

where $\dot{x}^{-}$is to be found from the equations of motion. This procedure produces the action

$$
\begin{aligned}
S=\int d \tau L^{\prime} & =\int d \tau \frac{p_{-}}{h}\left\{1-\sqrt{1-h v_{\perp}^{2}}\right\} \\
& =\int d \tau\left\{\frac{N_{p}}{2 R} v_{\perp}^{2}+\frac{15}{16} \frac{N_{p} N_{s}}{R^{3} M^{9}} \frac{v_{\perp}^{4}}{r^{7}}+\frac{225}{64} \frac{N_{p} N_{s}^{2}}{R^{5} M^{18}} \frac{v_{\perp}^{6}}{r^{14}}+\mathcal{O}\left(\frac{v^{8}}{r^{21}}\right)\right\}
\end{aligned}
$$


where $v_{\perp}^{2}=\dot{x}_{\perp}^{i} \dot{x}_{\perp}^{i}$. Note that all terms are linear in the momentum of the probe $N_{p}$.

This calculation, however, takes into account only the long distance interactions arising from the Einstein term $\sqrt{g} \mathcal{R}$ in the supergravity Lagrangian. At shorter distances, there are corrections to the Lagrangian involving higher order operators such as $\mathcal{R}^{4}$. Such corrections will yield additional terms plitude. The corrections can be derived by computing Feynman diagrams, or by a more convenient method which we now describe. Essentially, one needs to know how to pass from a field theory description of gravitons to a point particle description, so that the field theory corrections - like the presence of the $\mathcal{R}^{4}$ operator - can be translated into corrections to point particle actions which are easily compared with Matrix Theory results. The connection between field and particle actions is provided by Hamilton-Jacobi theory, as we now review in the simplified context of a scalar field theory.

Consider a massive scalar field

$$
\mathcal{L}=\frac{1}{2} \int d^{d} x \sqrt{g}\left(\partial_{\mu} \phi \partial^{\mu} \phi-m^{2} \phi^{2}\right) .
$$

Neglecting derivatives of the metric, the field equation is

$$
g^{\mu \nu} \partial_{\mu} \partial_{\nu} \phi+m^{2} \phi=0 \text {. }
$$

To pass to a point particle description, one uses the WKB form

$$
\phi=e^{i p_{\mu} x^{\mu}}
$$

which gives

$$
g^{\mu \nu} p_{\mu} p_{\nu}-m^{2}=0 .
$$

Now the idea is to solve for $p_{t}\left(p^{i}, m\right)$ and to regard $H=-p_{t}$ as the Hamiltonian of a point particle. Then one can work out the Lagrangian as usual

$$
L=p_{i} \dot{x}^{i}+p_{t}=-m \sqrt{g_{\mu \nu} \dot{x}^{\mu} \dot{x}^{\nu}} .
$$

One has thereby recovered the standard expression for the relativistic point particle action.

The action (9) was of only second order in derivatives, but one can easily add higher derivative terms, repeat the same procedure, and thus derive corrections to (13). It is this method which we now follow in the case of gravity.

\section{Calculations}

In this section we present the calculation of the effective action of a probe graviton moving in the classical background of a source graviton. We first show how to efficiently reproduce the result (8) starting from the Einstein action. Next, we extend the calculation to include

\footnotetext{
${ }^{1}$ Some discussion can be found in [20]. An $r^{-27}$ contribution has been discussed in [21].
} 
the higher derivative $\mathcal{R}^{4}$ operator. As we'll see, the operator produces a term in the probe action that does not seem to arise from any straightforward Matrix Theory calculation.

We begin with the eleven dimensional Einstein Lagrangian

$$
\mathcal{L}=\frac{1}{2 \kappa^{2}} \sqrt{g} \mathcal{R}
$$

We treat one of the two gravitons as a classical source which produces the metric (5). To this background, we add a small perturbation representing the presence of the probe graviton

$$
d s^{2}=d s_{\mathrm{bg}}^{2}+\kappa f_{\mu \nu} d x^{\mu} d x^{\nu}
$$

Instead of considering simultaneously all of the components of $f_{\mu \nu}$, we will choose a single, fixed, transverse and traceless polarization [2. We take

$$
f_{\mu \nu}=\left\{\begin{array}{l}
f_{12}\left(\tau, x^{-}, x^{3}, \ldots, x^{9}\right), \mu \nu=12,21 \\
0, \text { otherwise }
\end{array} .\right.
$$

Then, substituting (15) into the Lagrangian yields the following terms of second order in $f_{12}$

$$
\mathcal{L}=\frac{1}{4}\left\{-2 \partial_{\tau} f_{12} \partial_{-} f_{12}-\left(\partial_{\perp} f_{12}\right)^{2}+h\left(\partial_{\tau} f_{12}\right)^{2}\right\} .
$$

Note that now $\perp$ denotes the indices $i=3, \ldots, 9$. Now that we have a quadratic field action we can proceed as in sect. 2. The field equation is

$$
\left(-2 \partial_{\tau} \partial_{-}-\partial_{\perp}^{2}+h \partial_{\tau}^{2}\right) f_{12}=0
$$

We use the WKB form for $f_{12}$, and substitute

$$
f_{12}=e^{i p \cdot x}
$$

into (18). This gives an equation for $-p_{\tau}$, the Hamiltonian of the probe, as a function of $p_{-}, p_{\perp}, h$ :

$$
H=-p_{\tau}=\frac{p_{-}}{h}\left\{\sqrt{1+h p_{\perp}^{2} / p_{-}^{2}}-1\right\} .
$$

The probe action is then given as the $\tau$ integral of the Routhian $L^{\prime}$ constructed from (20):

$$
L^{\prime}=L-p_{-} \dot{x}^{-}=p_{i} \dot{x}^{i}+p_{\tau}=\frac{p_{-}}{h}\left\{1-\sqrt{1-h v_{\perp}^{2}}\right\}
$$

in agreement with the result in (8), but now derived in a way that readily admits generalization.

\footnotetext{
${ }^{2}$ As discussed in Appendix A, polarization dependence shows up through terms in the probe action which depend on derivatives of the harmonic function $h$. The present calculation correctly gives all the terms without derivatives.
} 
Now, we repeat this analysis in the presence of an $\mathcal{R}^{4}$ term arising from one-loop effects [11, 12]. The Lagrangian is

$$
\mathcal{L}=\frac{1}{2 \kappa^{2}} \sqrt{g} \mathcal{R}+\frac{c}{\kappa^{2 / 3}} \sqrt{g} t_{8} t_{8} \mathcal{R}^{4}
$$

where $c$ is a numerical coefficient argued to be [12]

$$
c=\frac{\pi^{2}}{9 \cdot 2^{7}}
$$

and $t_{8}$ is an 8 -index tensor, contracted with the Riemann tensor as follows:

$$
t_{8} t_{8} \mathcal{R}^{4}=t_{8}^{\mu_{1} \cdots \mu_{8}} t_{8}^{\nu_{1} \cdots \nu_{8}} \mathcal{R}_{\mu_{1} \mu_{2} \nu_{1} \nu_{2}} \cdots \mathcal{R}_{\mu_{7} \mu_{8} \nu_{7} \nu_{8}}
$$

For our perturbative considerations, we can follow the example of [12] and drop total derivative terms from (24); in this case the 8-index tensor $t_{8}$ is given explicitly by

$$
t_{8}^{\mu_{1} \cdots \mu_{8}}=\mathcal{S} \mathcal{A}\left\{24 \eta^{\mu_{2} \mu_{3}} \eta^{\mu_{4} \mu_{5}} \eta^{\mu_{6} \mu_{7}} \eta^{\mu_{8} \mu_{1}}-6 \eta^{\mu_{2} \mu_{3}} \eta^{\mu_{4} \mu_{1}} \eta^{\mu_{6} \mu_{7}} \eta^{\mu_{8} \mu_{5}}\right\}
$$

where $\mathcal{S}, \mathcal{A}$ are symmetrization and antisymmetrization operations on the indices, such that $t_{8}$ is antisymmetric in each pair of indices $\mu_{1}, \mu_{2} ; \mu_{3}, \mu_{4} ; \ldots$ and symmetric under the interchange of any pair of these index pairs (see [22]). These symmetry properties correspond to the symmetries of the Riemann tensor.

Next, we separate the total metric into a flat contribution and a correction term:

$$
d s^{2}=-2 d \tau d x^{-}-d x_{\perp}^{2}-h\left(d x^{-}\right)^{2}-\kappa f_{\mu \nu} d x^{\mu} d x^{\nu}=\left(\eta_{\mu \nu}+\Delta_{\mu \nu}\right) d x^{\mu} d x^{\nu}
$$

where

$$
\Delta_{\mu \nu}=-h \delta_{\mu-} \delta_{\nu-}-\kappa f_{\mu \nu}
$$

It would be an arduous task to try to find all corrections to the Routhian of the probe arising from the $\mathcal{R}^{4}$ term. We will instead focus on the leading term in the $1 / r$ expansion, which goes as $r^{-18}$, and is sufficient to demonstrate the apparent discrepancy with Matrix Theory. Let us consider first what sort of terms can give rise to $r^{-18}$ so that we know what we need to keep track of and what to neglect. Recalling $h \sim r^{-7}$, we quickly see that that the combination

$$
\partial \partial h \partial \partial h \sim \frac{1}{r^{18}}
$$

is the one that counts. Since $\mathcal{R}_{\mu \nu \rho \sigma}$ and $\mathcal{R}^{4}$ contain at most second derivatives of the metric, the terms of the type (28) are in fact the only ones that give $r^{-18}$ dependence. Thus many complicated terms can be dropped. For example, substituting $g_{\mu \nu}=\eta_{\mu \nu}+\Delta_{\mu \nu}$ into $\mathcal{R}_{\mu \nu \rho \sigma}$ yields

$$
\mathcal{R}_{\mu \nu \rho \sigma}=-\frac{1}{2}\left\{\partial_{\mu} \partial_{\rho} \Delta_{\nu \sigma}-\partial_{\nu} \partial_{\rho} \Delta_{\mu \sigma}-\partial_{\mu} \partial_{\sigma} \Delta_{\nu \rho}+\partial_{\nu} \partial_{\sigma} \Delta_{\mu \rho}\right\}+\cdots
$$


where $+\cdots$ denotes irrelevant terms which will not contribute to $\mathcal{O}\left(r^{-18}\right)$. Now, we can write out the $\mathcal{R}^{4}$ term in the Lagrangian and find

$$
c t_{8} t_{8} \mathcal{R}^{4}=c \cdot\left(2^{2}\right)^{4}\left(-\frac{1}{2}\right)^{4} t_{8}^{\mu_{1} \cdots \mu_{8}} t_{8}^{\nu_{1} \cdots \nu_{8}} \partial_{\mu_{1}} \partial_{\nu_{1}} \Delta_{\mu_{2} \nu_{2}} \cdots \partial_{\mu_{7}} \partial_{\nu_{7}} \Delta_{\mu_{8} \nu_{8}}+\cdots
$$

Then, substituting (27) and keeping terms with 2 factors of $\partial \partial h$ gives

$$
c t_{8} t_{8} \mathcal{R}^{4}=c \kappa^{2} \cdot 6 \cdot 2^{4} t_{8}^{i-k-\mu_{5} \cdots \mu_{8}} t_{8}^{j-l-\nu_{5} \cdots \nu_{8}} \partial_{i} \partial_{j} h \partial_{k} \partial_{l} h \partial_{\mu_{5}} \partial_{\nu_{5}} f_{\mu_{6} \nu_{6}} \partial_{\mu_{7}} \partial_{\nu_{7}} f_{\mu_{8} \nu_{8}}+\cdots .
$$

Note that (31) shows explicitly that the effect we are calculating is polarization dependent $t^{3}$. For the rest of the calculation we will fix the polarization of the graviton to be in the 12-plane, as in (16). Then, we find that

$$
\sqrt{g} \frac{c}{\kappa^{2 / 3}} t_{8} t_{8} \mathcal{R}^{4}=c \kappa^{4 / 3} K^{\mu \nu \alpha \beta} \partial_{\mu} \partial_{\nu} f_{12} \partial_{\alpha} \partial_{\beta} f_{12}
$$

where we have used $\sqrt{g}=1+\cdots$, since terms denoted by $+\cdots$ would only give terms that are higher order in $h$ or $f_{12}$, and we have introduced the tensor notation $K^{\mu \nu \alpha \beta}$ for

$$
K^{\mu \nu \alpha \beta} \equiv 6 \cdot 2^{5} \partial_{i} \partial_{j} h \partial_{k} \partial_{l} h\left\{t_{8}^{i-k-\mu 1 \alpha 1} t_{8}^{j-l-\nu 2 \beta 2}+t_{8}^{i-k-\mu 1 \alpha 2} t_{8}^{j-l-\nu 2 \beta 1}\right\} .
$$

Thus, the Lagrangian (22) becomes, to second order in $f_{12}$,

$$
\mathcal{L}=\frac{1}{4}\left\{-2 \partial_{\tau} f_{12} \partial_{-} f_{12}-\left(\partial_{\perp} f_{12}\right)^{2}+h\left(\partial_{\tau} f_{12}\right)^{2}\right\}+c \kappa^{4 / 3} K^{\mu \nu \alpha \beta} \partial_{\mu} \partial_{\nu} f_{12} \partial_{\alpha} \partial_{\beta} f_{12}
$$

In terms of the probe momenta, the field equation for $f_{12}$ isf

$$
-2 p_{\tau} p_{-}-p_{\perp}^{2}+h p_{\tau}^{2}+4 c \kappa^{4 / 3} K^{\mu \nu \alpha \beta} p_{\mu} p_{\nu} p_{\alpha} p_{\beta}=0
$$

Solving iteratively for $p_{\tau}$, we obtain

$$
p_{\tau}=p_{\tau}^{(0)}+\frac{2 c \kappa^{4 / 3}}{p_{-}}\left\{K^{\tau \tau \tau \tau}\left(p_{\tau}^{(0)}\right)^{4}+4 K^{\tau \tau \tau n}\left(p^{(0)}\right)^{3} p_{n}+\cdots\right\}
$$

where $p_{\tau}^{(0)}$ is the solution (20) found previously, and the index $n$ sums over - and the transverse coordinates $i$.

The final task is to perform the Legendre transformation to find the Routhian, and to restore the velocity $v_{\perp}$. Although all of the terms in the curly brackets in (36) will produce $r^{-18}$ dependent terms, only the first one contributes to a term of the form $v_{\perp}^{8} / r^{18}$, which has the velocity dependence we choose to focus on. In the Legendre transformation it is sufficient to use the approximation $p_{\tau}^{(0)} \approx-p_{\perp}^{2} / 2 p_{-}+\mathcal{O}(h)$ in the $\left(p_{\tau}^{(0)}\right)^{4}$ term. After some algebra we find the Routhian

$$
L^{\prime}=\frac{p_{-}}{h}\left\{1-\sqrt{1-h v_{\perp}^{2}}\right\}+\frac{c \kappa^{4 / 3}}{8} K^{\tau \tau \tau \tau} p_{-}^{3} v_{\perp}^{8}+\cdots
$$

\footnotetext{
${ }^{3}$ See also the discussion in Appendix A.

${ }^{4}$ Here we have used $\partial_{\mu} \partial_{\nu} K^{\mu \nu \alpha \beta}=K^{\mu \nu \alpha \beta} \partial_{\mu} \partial_{\nu}+\cdots$, where $+\cdots$ again denotes irrelevant terms.
} 
The coefficient of the last term can be found explicitly. Substituting (23), $p_{-}=N_{p} / R$, and from Appendix B,

$$
K^{\tau \tau \tau \tau}=6 \cdot 2^{5} \cdot \frac{7^{2} 4^{2} 15^{2} N_{s}^{2}}{R^{4} M^{18}} \frac{1}{r^{18}}
$$

and

$$
\kappa^{4 / 3}=\frac{4^{4 / 3} \pi^{10 / 3}}{M^{6}},
$$

we finally find that the last term in $L^{\prime}$ is

$$
14700 \pi^{5} \cdot(4 \pi)^{1 / 3} \cdot \frac{N_{s}^{2} N_{p}^{3}}{M^{24} R^{7}} \frac{v_{\perp}^{8}}{r^{18}} .
$$

As discussed in the introduction, the contribution (40) has the correct $v$ and $r$ dependence to match onto a two loop Matrix Theory term in (3), but the $N_{p}$ dependence is of the wrong form.

Although we have focussed on the $v^{8} / r^{18}$ contribution from the $\mathcal{R}^{4}$ term, there are other contributions as well whose forms we could easily derive. Clearly, by expanding $\sqrt{g} t_{8} t_{8} \mathcal{R}^{4}$ in different ways one can generate a wide variety of different contributions.

\section{Discussion}

It is useful to clarify, in the language of Feynman diagrams, which effects our calculation is including and which it is leaving out. In quantum mechanics language, we are doing

a calculation of potential scattering, in which one neglects the change in state of one of the scattered particles (the source) and uses only the classical potential produced by that particle. This is known as the effective field approximation; as discussed in [23] it is valid when the source particle is heavy and non-relativistic. In the case of gravitons in the light cone frame, this condition is $N_{s} \gg 1$ and $p_{\perp} \ll p_{-}$.

In our approach, we are writing the action as

$$
S\left(g_{\mu \nu}^{(\mathrm{cl})}+f_{\mu \nu}\right)
$$

and expanding to quadratic order in $f_{\mu \nu}$. Here $g_{\mu \nu}^{(\mathrm{cl})}$ is a classical solution to the action

$$
S\left(g_{\mu \nu}\right)+\int d^{11} x \sqrt{g} g_{\mu \nu} T^{\mu \nu}
$$

where $T^{\mu \nu}$ is the energy-momentum tensor of the source graviton. We could evaluate the metric seen by the probe by calculating

$$
\left\langle g_{\mu \nu}\right\rangle=\int \mathcal{D} g g_{\mu \nu} e^{i S\left(g_{\mu \nu}\right)+i \int \sqrt{g} g_{\mu \nu} T^{\mu \nu}}
$$

at the location of the probe. Keeping only the tree diagrams yields the classical metric, $\left\langle g_{\mu \nu}\right\rangle=g_{\mu \nu}^{(\mathrm{cl})}$. Pictorially, we are keeping diagrams like Fig. (2a) but discarding loop diagrams like Fig. (2b). 


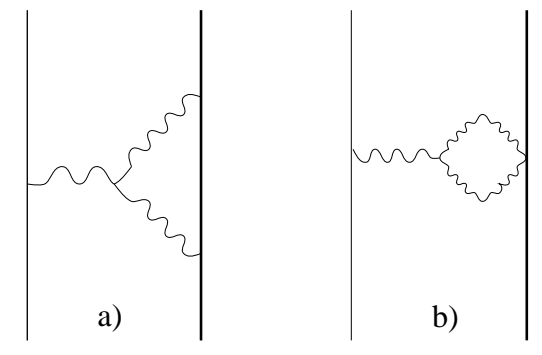

Figure 2: a) contribution corresponding to the classical metric of the source; b) quantum correction to the metric.

In treating the source graviton as a fixed classical metric we are neglecting recoil effects. This is valid provided that the emission of quanta by the source does not change its state appreciably. In other words, we require that the momentum exchanged in the scattering process should be small in comparison to the momentum of the source graviton.

In using only the classical value for the source metric $\left\langle g_{\mu \nu}\right\rangle$ we are neglecting certain correlation effects. That is, our calculation produces

$$
\left\langle g_{\mu \nu}(x) g_{\alpha \beta}(y)\right\rangle=\left\langle g_{\mu \nu}(x)\right\rangle\left\langle g_{\alpha \beta}(y)\right\rangle,
$$

and does not include corrections to this relation due to diagrams like that in Fig. 3.

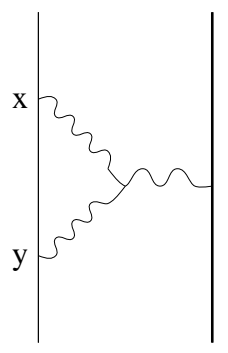

Figure 3: Diagram showing correlation of source fields on the probe.

Such processes correspond to non-local terms in the effective action for the probe and are not captured by our analysis.

Nowhere in our calculation did the anti-symmetric tensor or gravitino appear, and so one might be led to consider the possibility that the undesirable contribution we have found could be cancelled against diagrams involving the exchange of these fields. To see that this cannot happen, let us recall that the $\mathcal{R}^{4}$ arises from the divergent part of one loop diagrams, including the ones where the anti-symmetric tensor and gravitino circulate in the loop (see Fig. 1). Thus, at the level of two graviton exchange diagrams, we have already taken these fields into account insofar as their divergent effects are concerned. The only remaining contributions are the UV finite parts, but these have the property of being polynomial in $\kappa$, since non-polynomial dependence can only arise through the appearance of the UV cutoff. When we note that our result (2) contains a fractional power of $\kappa$, we conclude that it cannot be cancelled by the UV finite parts. Finally, in addition to $\mathcal{R}^{4}$ there are $\mathcal{R}^{5}$ and higher operators, but these can contribute only at the level of three or more graviton exchange diagrams, and so cannot yield a $r^{-18}$ dependence. 


\section{Conclusion}

We have seen that the presence of the higher dimension $\mathcal{R}^{4}$ operator in the low energy supergravity action leads to terms in the graviton-graviton scattering amplitude which have no simple analogues in Matrix Theory. The most likely resolution of the discrepancy is that we have been too naive in calculating within the context of supergravity with lightlike compactification. As discussed recently in [16], the presence of zero modes can complicate the dynamics of the lightlike compactified theory. In this case case, our supergravity results would not be valid in the domain in which they can be compared to finite $N$ Matrix Theory; instead, one should be comparing with the $N \rightarrow \infty$ limit. But in this limit one can conceive of effects which would invalidate the simple use of the effective action in the way that is normally done. It would then be important to determine which processes in Matrix Theory are protected from receiving $N \rightarrow \infty$ corrections so that they can be computed reliably at finite $N$.

\section{Acknowledgements}

We would like to thank Petr Hořava and John Schwarz for useful discussions and Senarath de Alwis for useful comments on an earlier version of this paper. The effect of the $\mathcal{R}^{4}$ operator has also been studied by Katrin and Melanie Becker [24].

\section{Appendix A}

Here make some comments on the polarization dependence of graviton scattering. Initially, one might worry about terms in the equation of motion for $f_{\mu \nu}$ which couple together different polarizations. However, the polarization dependent effects involve derivatives of $h$ and hence are subleading in $1 / r$. This observation is seen from the following argument. In flat space, the polarization does not affect the motion of the graviton. In curved space, one can always transform to locally flat coordinates, where space is flat up to subleading corrections. The polarization dependence must then come from the subleading terms. The transformation to locally flat coordinates, appropriate to the metric (5), is simply $\tau \mapsto \tilde{\tau}=\tau+h x^{-} / 2$, so the subleading corrections and the polarization dependence arise from derivatives of $h$.

However, with the inclusion of the $\mathcal{R}^{4}$ term the situation is somewhat different. Here the point is precisely to study the subleading corrections depending on derivatives of the harmonic function. Such corrections are expected to be polarization dependent, which is indeed the case as one can see from (31). In this paper we are only studying processes where the polarization remains fixed, which are simpler to handle.

\section{Appendix B}

Here we provide some details of the calculation of $K^{\tau \tau \tau \tau}$ which were skipped in section 4. The first step is to find explicit formulas for the components of $t_{8}$ which appear in the definition of $K^{\tau \tau \tau \tau}$. We proceeded as follows. First, we completed the antisymmetrization 
of $t_{8}$ as outlined in 22]. This results in a long expression with 60 terms, each involving a product of four metric factors $\eta^{\mu \nu}$. However, using

$$
\eta^{-\tau} \neq 0 ; \eta^{i i} \neq 0 ; \text { others }=0
$$

we get a much more compact equation for the following components of $t_{8}$ which appear in the definition of $K^{\mu \nu \alpha \beta}$ :

$$
\begin{aligned}
t_{8}^{i-k-\mu_{5} \cdots \mu_{8}}= & \eta^{i k}\left\{\eta^{-\mu_{5}}\left(\eta^{\mu_{6} \mu_{8}} \eta^{\mu_{7}-}-\eta^{\mu_{6} \mu_{7}} \eta^{\mu_{8}-}\right)\right. \\
& \left.-\eta^{-\mu_{6}}\left(\eta^{\mu_{5} \mu_{8}} \eta^{\mu_{7}-}-\eta^{\mu_{5} \mu_{7}} \eta^{\mu_{8}-}\right)\right\}
\end{aligned}
$$

Now we can evaluate $K^{\tau \tau \tau \tau}$. Recall that we defined $K^{\mu \nu \alpha \beta}$ by (33). Thus, the component $K^{\tau \tau \tau \tau}$ is given by

$$
K^{\tau \tau \tau \tau}=6 \cdot 2^{5} \partial_{i} \partial_{j} h \partial_{k} \partial_{l} h\left\{t_{8}^{i-k-\tau 1 \tau 1} t_{8}^{j-l-\tau 2 \tau 2}+t_{8}^{i-k-\tau 1 \tau 2} t_{8}^{j-l-\tau 2 \tau 1}\right\} .
$$

Using the result (44) above, we get

$$
\begin{aligned}
& t_{8}^{i-k-\tau 1 \tau 1}=\delta^{i k}\left(\eta^{-\tau}\right)^{2} \\
& t_{8}^{j-l-\tau 2 \tau 2}=\delta^{j l}\left(\eta^{-\tau}\right)^{2} .
\end{aligned}
$$

Similarly,

$$
t_{8}^{i-k-\tau 1 \tau 2}=t_{8}^{j-l-\tau 2 \tau 1}=0
$$

Substituting to (45), we find

$$
\begin{aligned}
K^{\tau \tau \tau \tau} & =6 \cdot 2^{5} \cdot \partial_{i} \partial_{j} h \partial_{i} \partial_{j} h \\
& =6 \cdot 2^{5} \cdot \frac{7^{2} 4^{2} 15^{2} N_{s}^{2}}{R^{4} M^{18}} \frac{1}{r^{18}} .
\end{aligned}
$$

In the last line, we substituted (6).

\section{References}

[1] T. Banks, W. Fischler, S.H. Shenker, L. Susskind, M theory as a matrix model: A Conjecture, Phys. Rev. D55, 5112 (1997), (hep-th/9610043). For a recent review, see T. Banks, Matrix Theory, hep-th/9710231.

[2] E. Cremmer, B. Julia and J. Scherk, Supergravity theory in eleven dimensions, Phys. Lett. B76 409 (1978).

[3] M.R. Douglas, D. Kabat, P. Pouliot and S.H. Shenker, D-branes and short distances in string theory, Nucl. Phys. B485, 85 (1997), (hep-th/9608024). 
[4] K. Becker and M. Becker, A Two loop test of M(atrix) theory, hepth/9705091.

[5] K. Becker, M. Becker, J. Polchinski, and A. Tseytlin, Higher order graviton scattering in M(atrix) theory, hep-th/9706072.

[6] J.A. Harvey, Spin dependence of D0-brane interactions, hep-th/9706039.

[7] J.F. Morales, C. Scrucca and M. Serone, A note on supersymmetric D-brane dynamics, hep-th/9709063.

[8] P. Kraus, Spin-orbit interaction from Matrix Theory, hep-th/9709199.

[9] I. Chepelev and A.A. Tseytlin, Long distance interactions of branes: correspondence between supergravity and super Yang-Mills descriptions, hepth/9709087.

[10] E. Keski-Vakkuri and P. Kraus, Born-Infeld actions from Matrix Theory, hep-th/9709122.

[11] M.B. Green and J.H. Schwarz, Supersymmetrical string theories, Phys. Lett. B109, 444 (1982).

[12] M.B. Green and P. Vanhove, D-instantons, strings and M-theory, Phys. Lett. B408, 122 (1997), (hep-th/9704145).

[13] M.B. Green, M. Gutperle, and P. Vanhove, One loop in eleven dimensions, Phys. Lett. B409, 177 (1997), (hep-th/9706175).

[14] J. Russo and A.A. Tseytlin, One loop four graviton amplitude in elevendimensional supergravity, hep-th/9707134.

[15] A. Sen, D0-Branes on $T^{n}$ and Matrix Theory, hep-th/9709220; N. Seiberg, Why is the Matrix Model correct?, hep-th/9710009; S.P. de Alwis, Matrix Models and string world sheet duality, hep-th/9710219.

[16] S. Hellerman and J. Polchinski, Compactification in the lightlike limit, hepth/9711037.

[17] L. Susskind, Another conjecture about M(atrix) theory, hep-th/9704080.

[18] M. Dine and A. Rajaraman, Multigraviton scattering in the Matrix Model, hep-th/9710174; M. Douglas and H. Ooguri, Why Matrix Theory is hard, hep-th/9710178; O.J. Ganor, R. Gopakumar and S. Ramgoolam, Higher loop effects in M(atrix) orbifolds, hep-th/9705188.

[19] P.C. Aichelburg and R.U. Sexl, On the gravitational field of a massless particle, Gen. Rel. Grav. 2, 303 (1971). 
[20] P. Berglund and D. Minic, A note on effective Lagrangians in Matrix Theory, hep-th/9708063.

[21] M. Serone A comment on $R^{4}$ coupling in M(atrix) Theory, hep-th/9711031.

[22] M.B. Green, J.H. Schwarz and E. Witten, Superstring theory, vol. 2, Cambridge University Press (1987), see Appendix 9.A.

[23] S. Weinberg, The quantum theory of fields, vol. 1, Cambridge University Press (1995), Section 13.6.

[24] K. Becker and M. Becker, to appear. 